\title{
Modification of Biodegradable Polyesters Using Electron Beam
}

\author{
M. Suhartini ${ }^{*}$ \\ Center for Application of Isotopes and Radiation Technology, National Nuclear Energy Agency \\ Jl. Lebak Bulus Raya No. 49, Jakarta 12440, Indonesia
}

\section{ARTICLE INFO}

Article history:

Received 20 November 2013

Received in revised form 27 December 2013

Accepted 31 December 2013

Keywords:

Poly(4-Hydroxybutirat)

Poly( $\varepsilon$-caprolactone)

Poly(butylene succinate-co-adipate)

Electron beam,

Polyfunctional monomer

\begin{abstract}
A B S T R A C T
Poly(4-Hydroxybutirat) P4HB, Poly(butylene succinate-co-adipate) PBSA and Poly( $\varepsilon$-caprolactone) PCL were electron beam (EB)-irradiated. Poly(4-Hydroxybutirat) was irradiated without any polyfunctional monomers (PFM). While PBSA and PCL were irradiated in the presence of polyfunctional monomers such as Trimethallyl isocyanurate (TMAIC), Polyethyleneglycol dimethacrylate $(2 \mathrm{G}, 4 \mathrm{G})$, Trimethylolpropane trimethacrylate (TMPT) and Tetramethylolmethane tetraacrylate (A-TMMT) at ambient temperature. Aim of the study is to improve the properties of biodegradable polyester. It was pointed out that crosslinking yield of P4HB $(6.39 \%$ gel) was formed at dose of $90 \mathrm{kGy}$ irradiated in vacuum conditions. Radiation degradation promoted, when P4HB was irradiated in air. The optimum crosslinking yield of PCL and PBSA respectively, were formed in the presence of $1 \%$ TMAIC at dose of $50 \mathrm{kGy}$. The biodegradability of the crosslinked PBSA evaluated by soil burial test is slightly retarded by increasing crosslinking yields.
\end{abstract}

(C) 2013 Atom Indonesia. All rights reserved

\section{INTRODUCTION}

Biodegradable polyesters have been widely used in many industrial applications. How ever some biodegradable polyesters have relatively low melting point, low molecular weight and poor processability, which limit the industrial applications [1]. Irradiation processing is acknowledged to be one of the most important technology for polymer macromolecules crosslinking. It is a convenient tool for modification of the polymeric materials via crosslinking, grafting and degradation [2]. By crosslinking of polymer, their chemical, physical, and mechanical properties can be improved to a great extent.

The most important chemical changes that occur during the irradiation of polymers are those of crosslinking and degradation. When solid polymeric materials are exposed to ionizing radiation, crosslinking or degradation may occurred depending mainly on their chemical nature and also irradiation conditions. Both processes take place simultaneously in most polymer systems. The one which predominates determines the net effect. The polymer tends to crosslink if each carbon atom of the main chain carries at least one hydrogen atom (Fig. 1). While degradation will predominantly occur in the polymer with tetra substituted carbon in

\footnotetext{
* Corresponding author.

E-mail address: meri@batan.go.id
}

the repeating unit (Fig. 2) [3]. For some polymers such as [poly(vinyl chloride), polypropylene, poly(ethylene terephthalate)] both direction of transformations are possible to occur. A change in irradiation conditions may shift a polymer from one group to the another. For instance, polyvinyl alcohol usually undergoes degradation, but it is crosslinked in the presence of water [3]. Polycarbonate behaves as a crosslinking polymer at low doses $(20 \mathrm{kGy})$ and as degrading polymer at high dose (100 kGy) [4].<smiles>[R]CCC</smiles>

Fig. 1. Crosslinking polymer<smiles>[R]C([R4])([R])C(C)CC</smiles>

Fig. 2. Degrading polymer.

Aliphatic polyesters such as Poly(4Hydroxybutirat) P4HB, Poly(butylene succinate-coadipate) PBSA, Poly( $\varepsilon$-Caprolactone) PCL, Poly(Lactic Acid) PLA, are environmentally friendly polymers because they are able to be converted to water and carbon dioxide under the action of degradation reaction by microorganisms in 
the soil. However, the insufficient properties of these polymers (i.e., deformation in boiling water) prevent them from being utilized in diverse applications. Hence improvement of their heat stability is desired. Irradiation processing in supercooled state can improve heat resistance and process ability of Poly(E-caprolactone) (PCL) [4]. Poly(butylene succinate) PBS has been studied to produce a biodegradable foam by electron beams irradiation [5]. The useful products of polymer could be produced only if certain additives were incorporated into polymer. With the help of suitable additives, the heat stability, mechanical properties and process ability of plastic can be improved [6]. It was investigated that using irradiation or/and a specific monomer distribution in the polymer chains of aliphatic polyesters, new polymeric materials with useful properties can be synthesized. Specific monomer distribution in the polymer chains of aliphatic polyesters formed good material properties with a reasonable price level and biodegradability [7]. The physical properties of aliphatic polyesters were improved as a result of addition of aromatic units into the polymer [8]. It is known that the biodegradation rate of PBS strongly depends on degree of its crystallinity and morphology. Aliphatic polyesters with aromatic groups in their side chain should have properties and biodegradability different from those with aromatic groups in their main backbone chains [9]. To improve the properties of alipathic polyesters by irradiation processing is required to know the effect of irradiaton dose and monomer addition on properties and degradability of aliphatic polyesters. Therefore, in this work, radiation crosslinking of $\mathrm{P} 4 \mathrm{HB}$ without any monomer and PCL and PBSA blended with certain polyfunctional monomers was investigated.

\section{EXPERIMENTAL METHODS}

\section{Materials}

P4HB with $90 \%$ concentration and average molecular weights $4.7 \times 10^{4}$ was made in biological and chemical engineering laboratory of Gunma University - Japan. PCL and PBSA with average molecular weights $2.19 \times 10^{5}$ and $2.96 \times 10^{5}$ respectively (Table 1 ) were produced at Showa High Polymer Co. Ltd., Japan. Five polyfunctional monomers used in this work are as follows: Trimethallyl isocyanurate (TMAIC), Polyethyleneglycol dimethacrylate (2G, 4G), Trimethylolpropane trimethacrylate (TMPT) and Tetramethylolmethane tetraacrylate (A-TMMT). The structure of polyfunctional monomer used in this work is shown in Table 2.

Table 1. Properties of polyester samples used in this work.

\begin{tabular}{lccccc}
\hline Sample type & Mw & Mw/Mn & $\begin{array}{c}\text { Melting } \\
\text { point }\left({ }^{\circ} \mathrm{C}\right)\end{array}$ & $\begin{array}{c}\text { Density } \\
\left(\mathrm{g} / \mathrm{cm}^{3}\right)\end{array}$ & $\begin{array}{c}\triangle \mathrm{H} \\
(\mathrm{J} / \mathrm{g})\end{array}$ \\
\hline $\begin{array}{l}\text { P4HB } \\
\text { Poly(4-Hydroxybutirat) }\end{array}$ & $4.2 \times 10^{4}$ & 2,8 & 63 & 1.213 & 17 \\
\hline $\begin{array}{l}\text { PCL } \\
\text { Poly(E-Caprolactone) }\end{array}$ & $2.19 \times 10^{5}$ & 1.53 & 60 & 1.145 & 44 \\
\hline $\begin{array}{l}\text { PBSA } \\
\begin{array}{l}\text { Poly(butylene } \\
\text { succinate-co-adipate) }\end{array}\end{array}$ & $2.96 \times 10^{5}$ & 2.0 & 92 & 1.230 & 45 \\
\hline
\end{tabular}

Table 2. Structure of polyfunctional monomer used in this work.

\begin{tabular}{l}
\hline Name \\
$\begin{array}{l}\text { Primethallylisocyanu (TMAIC) } \\
\text { methacrylate (2G) }\end{array}$ \\
$\begin{array}{l}\text { Polyethyleneglycol dimethyl } \\
\text { methacrylate (4G) }\end{array}$ \\
$\begin{array}{l}\text { Trimethylolpropane trimethac } \\
\text { (TMPT) }\end{array}$ \\
$\begin{array}{l}\text { Tetramethylolmetthane tetra (A- } \\
\text { TMMT) }\end{array}$
\end{tabular}




\section{Preparation of samples and irradiation}

$P 4 H B$

Sintesis $\mathrm{P} 4 \mathrm{HB}$ was done using $D$. Acidovorans bacteria through microorganisms fermentation. The fermentation conditions are : nutrient-rich medium (pH 7.0; 1L) containing $10 \mathrm{~g}$ polypeptone, $10 \mathrm{~g}$ of yeast extract, $5 \mathrm{~g}$ of meat extract and $5 \mathrm{~g}\left(\mathrm{NH}_{4}\right)_{2} \mathrm{SO}_{4}$ fermented at $26^{\circ} \mathrm{C}$ for 48 hours in a reactor. Subsequently cells were taken after 48 hours and washed with water. To improve the synthesis of polyesters, the washed cells was transferred to mineral medium ( $\mathrm{pH}$ 7.0) containing different carbon substrates. Cells were grown in this medium at $26^{\circ} \mathrm{C}$ for 72 hours, then centrifuged $(4000 \mathrm{rpm}$, $15 \mathrm{~min}$ ), washed with water, methanol and then dried using a vacuum at room temperature. Polyester was extracted from dried cells using hot chloroform and purified by precipitation using $n$ heptane [10]. The P4HB polymer was then dried with vacuum for 48 hours. Irradiation of samples was carried out in vacuum plastic bags and under air using an accelerator with energy of $2 \mathrm{MeV}$ and current of $1 \mathrm{~mA}$, at various doses, ranging from 45 to $200 \mathrm{kGy}$ at a dose rate of $10 \mathrm{kGy} /$ pass.

\section{$P C L$ and $P B S A$}

PCL or PBSA and the polyfunctional monomer were mixed in a labo plastomill model $50 \mathrm{C} 150$ (Toyo Seiki). The concentration of the polyfunctional monomer was $0,2 \% ; 0,5 \% ; 1 \%$ and $3 \%$ weight. The speed of mixing was $20 \mathrm{rpm}$, at $150^{\circ} \mathrm{C}$. Each sample was pressed to form $0.5 \mathrm{~mm}$ thickness sheet in an Ikeda hot press at $150^{\circ} \mathrm{C}$, samples were preheated for $3 \mathrm{~min}$, then were heated at the same temperature for another $3 \mathrm{~min}$ at a pressure of $120 \mathrm{kgf} / \mathrm{cm}^{2}$. Irradiation of samples was carried out in vacuum plastic bags using an accelerator with $2 \mathrm{MeV}$ energy and current of $1 \mathrm{~mA}$, at various doses, ranging from 10 to $100 \mathrm{kGy}$ at a dose rate of $10 \mathrm{kGy} /$ pass.

\section{Thermal analysis measurement}

Melting temperatures were measured using differential scanning calorimeter (DSC-7, Perkin Elmer) with a heating or cooling rate $20^{\circ} \mathrm{C} / \mathrm{min}$.

\section{Gel fraction measurements}

The gel content of irradiated polyesters was analyzed after removing the soluble part by extraction in boiling chloroform for 48 hour.

\section{Soil burial test}

The soil burial test was performed in the plastic box $57 \mathrm{~cm}$ long, $17 \mathrm{~cm}$ wide and $14 \mathrm{~cm}$ high. The dumbbell cut samples with a thickness of $0.5 \mathrm{~mm}$ were buried in the soil in about $3 \mathrm{~cm}$ depth at various time between 1 and 6 months.

\section{RESULTS AND DISCUSSION}

\section{Crosslinking behaviors of P4HB}

As shown in Fig. 3 gel fraction of poly (4-hydroxybutyrate) as a function of irradiation dose, it can be seen that at irradiation dose of $90 \mathrm{kGy}$ under vacuum, gels of $6.39 \%$ was formed then increased linearly with increasing dose of irradiation. At irradiation dose of $200 \mathrm{kGy}$ Gels of $27.19 \%$ are formed. Whereas no gel formation for irradiation under air at room temperature. The electron beam generate heat energy during the irradiation time. In vacuum irradiation condition, the temperature of polyester increased above its melting point, due to electron beam heat energy and low air pressure. The polyesters contain crystalline and amorphous parts at room temperature, crosslinking is hardly formed in the crystalline phase. At temperature above melting point, the crystalline of polyesters is converted to amorphous state [11], how ever easier to be croslinked by irradiation. Irradiation in vacuum condition causes increasing temperature higher than that in air.

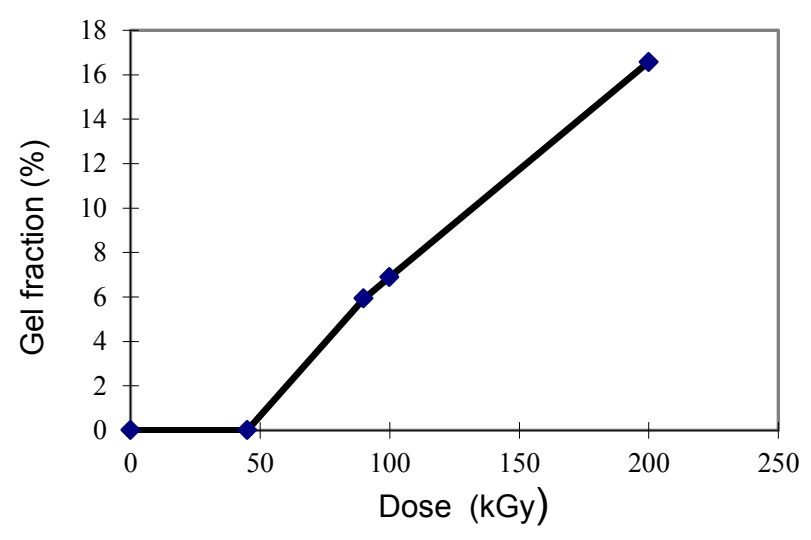

Fig. 3. Gel fraction dose curves of P4HB.

Crosslinking and chain scissions are among the effects observed when polymers are subjected to irradiation. The processes ultimately cause formation of an insoluble gel if crosslinking predominates over scission. Poly(4hydroxybutyrate) is a crosslinkable polymer, as evidenced by the formation of gel when exposed to electron beams. 
Generaly, the formation of a crosslink requires the presence of two neighbouring radicals, one on each polymer chain [11]. It is postulated that in solid polymers radicals can move and decay slowly. Such mobility can be achieved by a series of hydrogen abstraction and addition reactions. The excited polymer molecule may be produced either by direct excitation or by the result of recombination between polymer radical cation and thermalized electron. After absorption of the energy in polymer material, the resultant neutral excited molecule can release energy excess by dissociation into free radicals by bond cleavage in main or side-chain or by abstraction of $\mathrm{H}$ atom. Abstracted side-chain radicals or hydrogen atoms have a higher mobility than polymeric radicals and may have sufficient excess of kinetic energy to be transported some distance from its origin. In that case they can react with another macromolecule being the cause of second macroradical creation. Finally, when two polymer chain radicals are situated in their vicinity they may recombine and form a new covalent, crosslinked bond. The mechanism of radiation crosslinking of polymers can be described as follows [12].

(polymer) $\mathrm{PH} \rightarrow h v \rightarrow \mathrm{PH}^{*}$

$\mathrm{PH} \rightarrow h v \rightarrow \mathrm{PH}^{+}+\mathrm{e}^{-} \rightarrow \mathrm{PH}^{*}$

$\mathrm{PH}^{*} \rightarrow \mathrm{P}^{\bullet}+\mathrm{H}^{\bullet}$

$\mathrm{PH}^{+}+\mathrm{PH} \rightarrow \mathrm{P}^{\bullet}+\mathrm{PH}_{2}^{+}$

$\mathrm{P}^{\bullet}+\mathrm{PH}_{2}^{+}+\mathrm{e}^{-} \rightarrow \mathrm{P}^{\bullet}+\mathrm{P}^{\bullet}+\mathrm{H}_{2}$

$\mathrm{P}^{\bullet}+\mathrm{P}^{\bullet} \rightarrow \mathrm{P}-\mathrm{P} \quad$ (crosslinked polymer)

Degradation or crosslinking is often accompanied by gas evolution. The principal gas evolved from hydrogen-containing polymers is usually hydrogen, although other gases sometimes predominate. Gases may be released as by-products of crosslinking reaction, or they may derive from side-chain decomposition or main-chain unsaturation not associated with crosslinking.

In the presence of oxygen, which produces peroxides, hyperperoxides tends to accelerate the breakdown of some polymers when exposed to ionizing radiations, the reactions can be described as follow [12]:

(polymer) $\mathrm{PH} \rightarrow h v \rightarrow{ }^{\circ} \mathrm{P}+{ }^{\bullet} \mathrm{H}$

$\cdot \mathrm{P}+\mathrm{O}_{2} \rightarrow \mathrm{POO}^{\bullet}$

$2 \mathrm{POO}^{\bullet} \rightarrow$ POOOOP $\rightarrow 2 \mathrm{PO}^{\bullet}+\mathrm{O}_{2}$

$\mathrm{PO}^{\bullet} \rightarrow{ }^{\bullet} \mathrm{P}_{\mathrm{a}}+{ }^{\bullet} \mathrm{P}_{\mathrm{b}}$ (degraded polymer)

\section{Crosslinking behaviors of PCL and PBSA}

The gel contents of PCL and PBSA in the presence of different $1 \%$ weight monomers (TMAIC, $3.44 \times 10^{-3} \mathrm{~mol} \%$; A-TMMT, $2.84 \times 10^{-3}$ mol\%; TMPT, $2.96 \times 10^{-3} \mathrm{~mol} \%$; $2 \mathrm{G}, 4.13 \times 10^{-3}$ $\mathrm{mol} \%$; 4G, $3.03 \times 10^{-3} \mathrm{~mol} \%$ ) versus doses of irradiation are shown in Figs. 4 and 5 respectively.

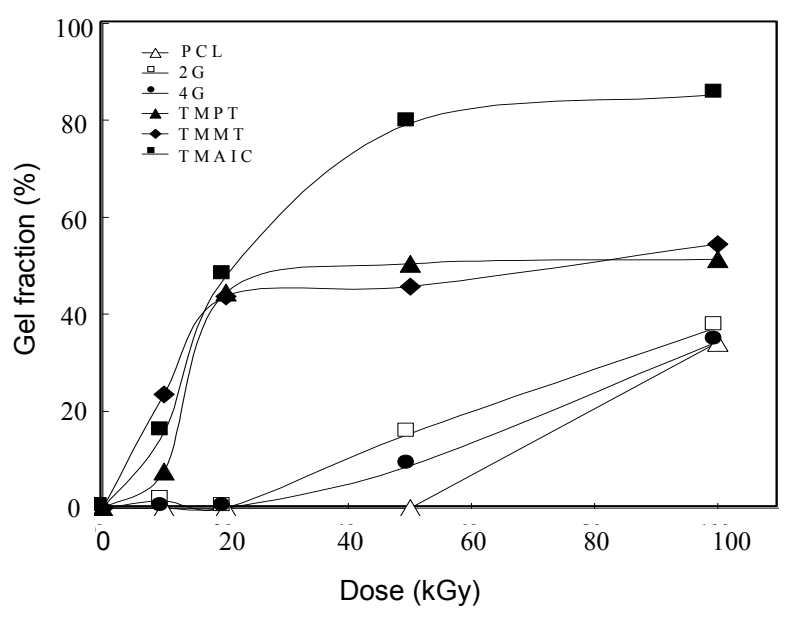

Fig. 4. Gel fraction dose curves of PCL containing 1\% weight of different monomers.

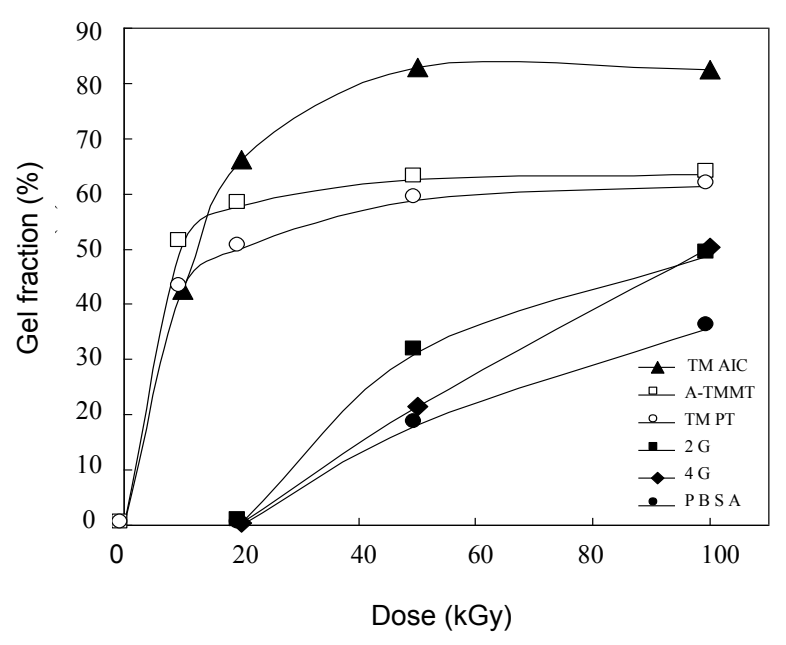

Fig. 5. Gel fraction-dose curves of PBSA containing 1\% weight of different monomers.

It can be seen that there is no gel formed for irradiated pure PCL whereas irradiated pure PBSA reaches only $18 \%$ of gel formed at $50 \mathrm{kGy}$ of irradiation dose. For mixed samples, however, the gel formed increases significantly with an addition of PFMs. The gel fractions are $79 \%$ for PCL and $83 \%$ for PBSA, by adding $1 \%$ TMAIC at the same irradiation dose above. The addition of TMAIC gave a rapid increase of gel formation of both PCL and PBSA. The irradiation 
dose reached the optimum at $50 \mathrm{kGy}$. The addition of TMAIC resulted higher value of formed gel compared with other PFM; A-TMMT, TMPT, $2 \mathrm{G}$ and $4 \mathrm{G}$ either for PCL or PBSA. The formed gels by adding $1 \%$ TMAIC were $79 \%$ for PCL and $83 \%$ for PBSA at irradiation dose of $50 \mathrm{kGy}$. Trimethallyl isocyanurate has a cyclic unit that achieves a greater three-dimensional network by irradiation than that of acrylate polyfunctional monomers molecules. It is well known that when bulk polymers are irradiated, the dominant reactions and the final products depend strongly on the transport of excitation energy (and charge), strengths of individual chemical bonds, and on the geometrical arrangements of the polymer molecules. Usually, in most polymers one of the two general processes dominates, either crosslinking or degradation [11].

Figure 6 shows the gel content of irradiated PCL containing different amounts of TMAIC. It can be seen that irradiated PCL containing $0.5 \%$, and $1 \%$ TMAIC form higher gel content than that of PCL containing 3\% TMAIC at the same irradiation doses. Similar behavior was found for irradiated PBSA containing TMAIC at dose of 10 and $20 \mathrm{kGy}$ (Fig. 7). Where as at irradiation dose of $100 \mathrm{kGy}$, PBSA containing $2 \%$ and 3\% TMAIC have higher gel content than that of PBSA containing $0.2 \%, 0.5 \%$ and $1 \%$. This is probably because PBSA produces sufficiently high amount of polymer radicals that react with monomers at a dose of $100 \mathrm{kGy}$. At this condition, all monomers can be used to initiate an effective crosslinking in the polymer. The optimum gel fraction was formed in the presence of $1 \%$ TMAIC at dose of $50 \mathrm{kGy}$ for both PCL and PBSA.

The different situation arises when crosslinks occur via a chain reaction, involving both polymer and monomer. For example is the curing of unsaturated polyester/styrene mixtures. Here, the initiation of a chain reaction via a single radical allows a number of crosslinks to occur in a sequence. Furthermore, the dose requirements are considerably reduced. For this purpose one double bond per monomer unit is needed to propagate the chain and in addition one double bond per crosslink to allow the growing polymer chain to attach itself to the polyester. This extra double bond can either occur in the monomer or on the polymer chain. Thus the average overall number of unsaturated groups per monomer reacted must exceed unity. Not only the dose requirements considerably reduced, but the relationship between solubility and crosslinked density is greatly modified.

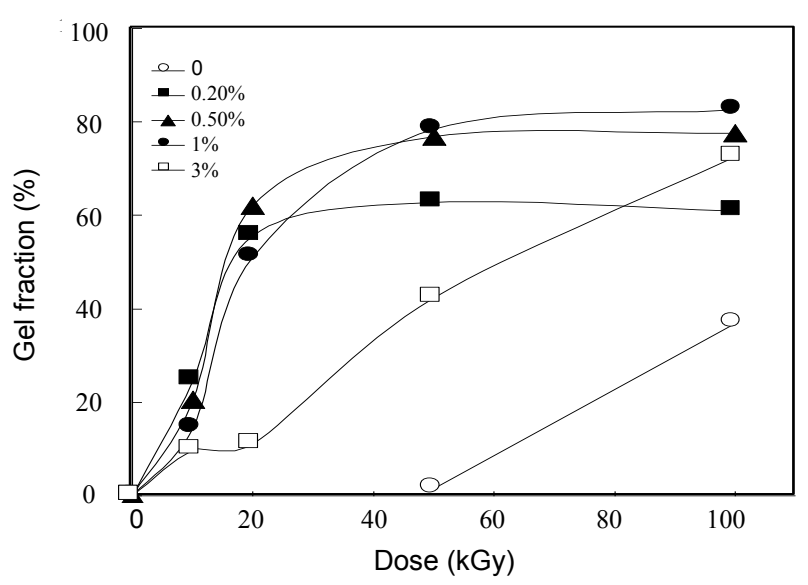

Fig. 6. Gel fraction-dose curves of PCL with TMAIC in different concentrations.

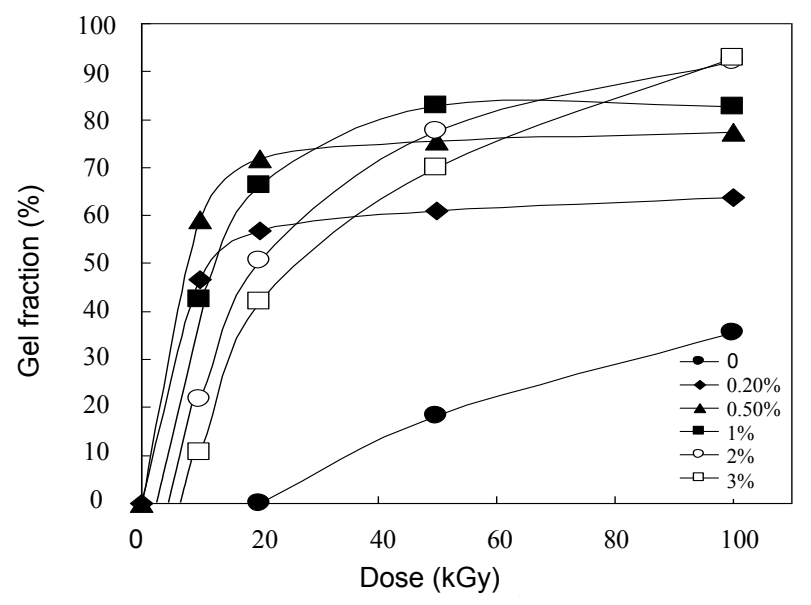

Fig. 7. Gel fraction-dose curves of PBSA with TMAIC in different concentrations.

\section{Melting behaviors of crosslinked P4HB}

Figure 8 shows melting behaviors curves of $\mathrm{P} 4 \mathrm{HB}$ and its crosslinked form at dose of $90 \mathrm{kGy}$ $(6.39 \%$ gel $)$ and $200 \mathrm{kGy} \quad(27.19 \%$ gel $)$ respectively. It can be seen that the peak temperatures of endotherms decreased from $63^{\circ} \mathrm{C}$ (P4HB without gel) to $59^{\circ} \mathrm{C}$ (P4HB with $6.39 \%$ gel). Furthermore the peak temperatures of endotherms (melting point) of irradiated P4HB contains 27.19\% gel, became broader and decreased to $53^{\circ} \mathrm{C}$. These behaviors of irradiated P4HB can be explained as follow: The melting temperature is often discussed on the basis of crystallite size so that decrease in melting temperature indicates decrease in crystallite size [13]. Decrease in crystallite size without change in distortion parameter and unit cell dimension possibly suggests that crystals are degraded in the vicinity of the crystalline-non-crystalline interface. Crystallization temperature becomes broader and shifts to lower temperature with increasing gel content as shown in Fig. 8. It is understood that 
crosslinking imparts a remarkable influence on crystallization.

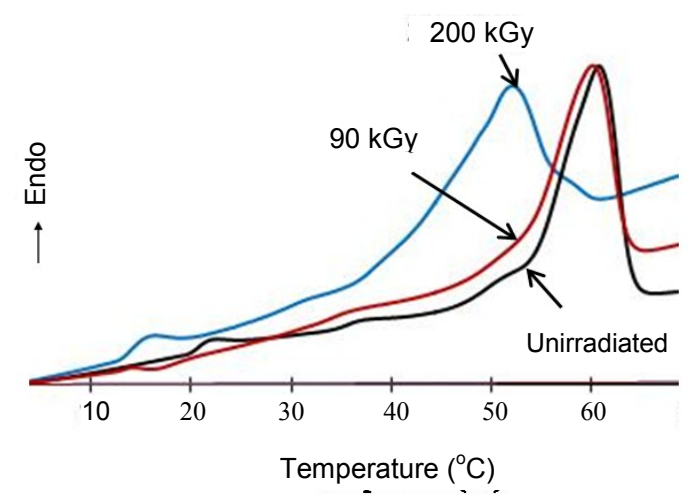

Fig. 8. Melting behaviors curves of irradiated P4HB.

\section{Melting behaviors of crosslinked PBSA}

The presence of TMAIC in PBSA samples enhanced the yield of gel formation by irradiation. To elucidate the mechanism of irradiated PBSA in the presence of TMAIC, an effect of crosslinking yield on sample's morphology was investigated. Figure 9 shows melting behaviors curves of PBSA and its crosslinked form. The DSC second rund heating was performed directly after the cooling of the first run. The melting point of PBSA sample in second run slightly lower than in the first run due to short time available to form crystalline, so that the crystallization is not completed. Increasing of crosslinking yield depresses the melting point of PBSA, as shown in Fig. 9. Crystallization temperature shifts to lower temperature with increasing gel content as shown in Figure 9. It is understood that crosslinking imparts a remarkable influence on crystallization.

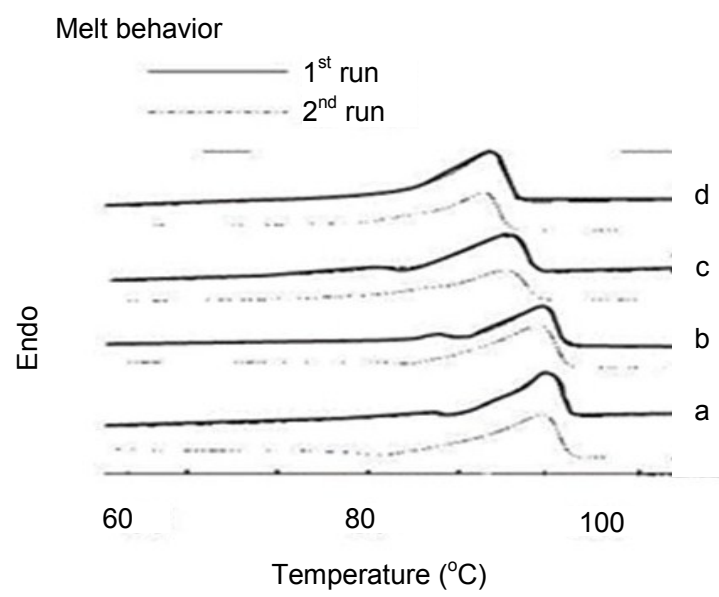

Fig. 9. Melting behaviors curves of irradiated PBS1 in the presence of $1 \%$ of TMAIC: (a) $0 \%$ gel fraction, (b) $43 \%$ gel fraction, (c) $83 \%$ gel fraction and (d) $100 \%$ gel fraction.

Figure 10 shows the enthalpy of PBSA against gel content, one can see a decrease in the sample enthalpy when the gel content is increased. The enthalpy of polymer depends on the amount of crystallite. Evidently, increasing the yield of gel formation decreased the amount of crystallite in PBSA samples.

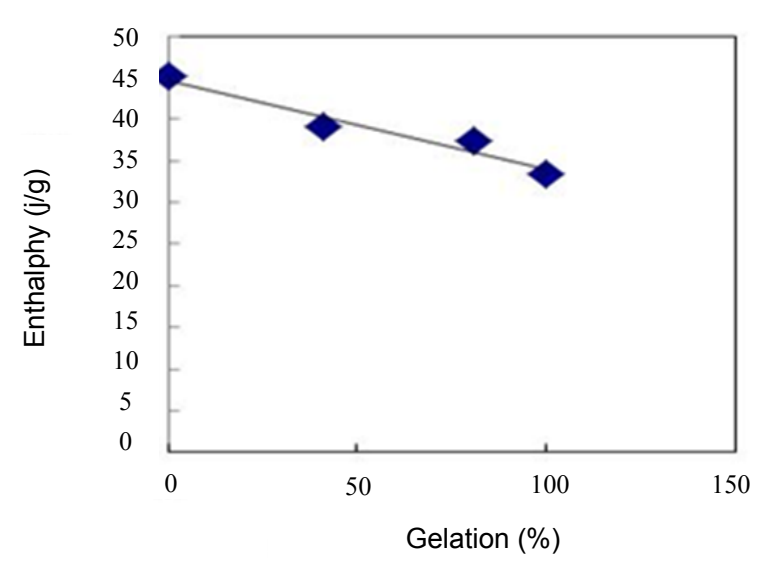

Fig. 10. Enthalpy-gel fraction curves for PBSA.

\section{Soil burial test}

Soil burial test was conducted to elucidate the effect of crosslinking yield on the biodegradability of polyester. Biodegradation occurs with enzymatic action and involves living organisms (micro/macro). Molecular degradation is promoted by enzymes and can occur under aerobic and anaerobic conditions, leading to complete or partial removal from the environment. Linear polymers are generally more biodegradable than branched polymers. The biodegradability of PBSA in diferent crosslinking yield has been examined in the soil. The soil burial biodegradation of a material depends on the temperature, humidity, $\mathrm{pH}$, oxygen concentration, insufficient number of active microorganism, availability of mineral nutrient and low glass temperature (high mobility) etc. Soil burial test is a standard examination of biodegradability of polymer samples by bacteria in soil.

Usually, enzymatic degradation of polyesters is a surface erosion process, because enzymes are not able to penetrate the polymer. Thus the weight loss can be used to measure the enzymatic cleavage of the polymer. The crosslinking yield of polyester can be indicated by the gel content in the polyester. The weight loss results presented in Fig. 11. It can be seen that weight loss of irradiated PBSA containing $1 \%$ TMAIC with $83 \%$ gel slightly diminishes compared to irradiated pure PBSA with $26 \%$ gel which irradiated at the same dose with PBSA containing 1\% TMAIC. While unirradiated PBSA $(0 \%$ gel) degraded faster than those of 
irradiated PBSA with $83 \%$ gel and $26 \%$ gel. The rate of enzymatic degradation decreases with an increase in glass temperature.

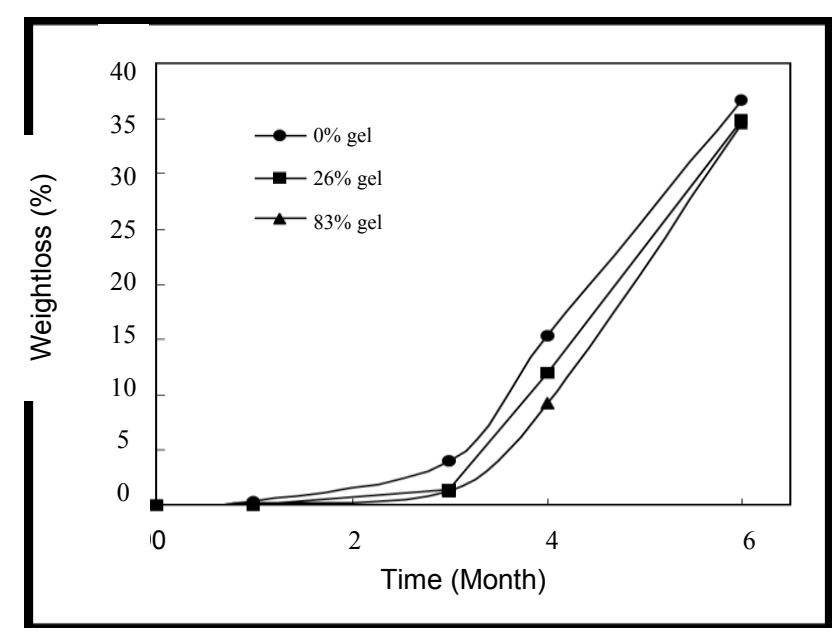

Fig. 11. Soil burial test of PBSA with different gel contents (pure PBSA $(0 \%$ gel); irradiated PBSA $(26 \%$ gel) irradiated at $50 \mathrm{kGy}$; irradiated PBSA containing 1\% TMAIC (83\% gel) irradiated at $50 \mathrm{kGy}$ ).

\section{CONCLUSION}

Irradiated P4HB formed 6,39\% gel, while irradiated PCL and PBSA formed more than 30\% gel respectively at irradiation dose of $90 \mathrm{kGy}$. Poly(4-Hydroxybutyrate) P4HB has less tendency to crosslink compare to PCL and PBSA. The melting temperature of P4HB and PBSA decreased by increasing irradiation dose. Increasing the yield of gel formation decreased the amount of crystallite. Trimethallyl isocyanurate stronger enhanced formations of gel in the irradiated PBSA or PCL compared to the other polyfunctional monomers. The amount of TMAIC blended with PBSA or PCL influences the amount of gel resulting after irradiation. The most efficient crosslinking took place for PBSA or PCL which contains 1\% TMAIC at irradiation dose of $50 \mathrm{kGy}$. Increasing of crosslinking in PBSA caused retarding of biodegradability.

\section{ACKNOWLEDGMENT}

The author would like to thank to JAEA and Gunma University for the permission to use the irradiation facilities, providing the chemicals and the scientific equipments. The author is also grateful to Dr. F. Yoshii (JAEA) and Prof. Dr. H. Mitomo (Gunma University) for his kind help and suggestions in this work.

\section{REFERENCES}

1. E. Takiyama and T. Fujimaki, Biodegradable plastics and polymers, Elsevier, Amsterdam, (1994) 150.

2. E.I. Grigoriev and L.I. Trakhtenberg, Radiation chemical processes in solid phase: theory and application, CRC Press, Boca Raton, New York, London, Tokyo (1996) 179.

3. A. Miller, E.J. Lawton and J.S. Balwit, J. Polym. Sci. 14 (1954) 503.

4. F. Yoshii, D. Darwis, H. Mitomo and K. Makuuchi, Radiat. Phys. Chem. 57 (2000) 417.

5. K. Bahari, H. Mitomo, T. Enjoji, F. Yoshii and K. Makuuchi, Polym. Degrad. Stab. 62 (1998) 551.

6. U. Witt, R-J. Muller and W-D. Deckwer, J. Macromol. Sci. Pure. Appl. Chem. A 32(4), (1994) 851.

7. U. Witt, R-J. Muller, J. Augusta, H. Widdecke and W-D. Deckwer, Macromol. Chem. Phys. 195 (1994) 793.

8. Y. Tokiwa and T. Suzuki, J. Appl. Polym. Sci. 26 (1981) 441.

9. H.J. Jin, B.Y. Lee, M.N. Kim and J.S. Yoon, Eur. Polym. J. 36 (2000) 2693.

10. C. Hsieh Wen, Poly(3-Hydroxybutyrate-co-4Hydroxybutyrate) biosiynthesized by D. Acidovorans and its physical properties, Ph.D. Thesis, Gunma University (2003).

11. Charlesby, Radiat. Phys. Chem. 59 (1981) 12.

12. T.S. Ikitina, E.V. Zhuravskaya and A.S. Kuzminsky, Effect of Ionizing Radiation on High Polymers, Gordon and Breach Inc., New York (1963).

13. L. Mandelkern, Crystallization of polymers, McGraw-Hill, New York (1964). 